\title{
Comparison of hemodynamics in post-operation coarctation of aorta patients with different aortic arch geometry
}

\author{
Qiong Yao ${ }^{1,2}$, Lili Pan ${ }^{1}$, Quanli Shen ${ }^{1,2}$, Zhongwei Qiao ${ }^{1}$, Floris-Jan S Ridderbos ${ }^{3}$, Xihong Hu ${ }^{1,2, *}$ \\ ${ }^{1}$ Department of Radiology, Children's Hospital of Fudan University, 201102, Shanghai, China; ${ }^{2}$ Pediatric Heart \\ Centre, Children's Hospital of Fudan University, 201102, Shanghai, China; ${ }^{3}$ Center for Congenital Heart Disease, \\ Department of Paediatric Cardiology, Beatrix Children's Hospital, University of Groningen, Groningen, the Netherlands
}

*corresponding author: Xihong Hu, Tel: 86-21-64931805, Fax: 86-21-64931901, E-mail: huxihong @ gmail.com

Received May 28, 2019; Accepted September 11, 2019

Aim: Coarctation of the aorta (COA) is associated with post-operation deformation and abnormal hemodynamics. The objective of this work is to quantify hemodynamic alterations with different vascular structures of post-operation CoA with computational fluid dynamics techniques.

Methods: A total of 4 patients with different aortic shapes were included in the research: No.1=no stenosis; No.2=slight stenosis (stenosis/descending $=78.3 \%$ ); No.3=moderate to severe stenosis (stenosis/descending= $55.7 \%$ ) and No.4 = angular aortic arch. MR axial images were adopted and phase-contrast sequence provided the inlet velocity-time curve. A three-dimensional model for simulation was modeled by Mimics 17.0 and Fluent 15.0 was applied to simulate the blood flow within the thoracic aorta. Measurements of relevant hemodynamic parameters included streamline, wall pressure and wall shear stress (WSS).

Results: The hemodynamic parameters of thoracic aorta at three-time phases were demonstrated. Compared to the patient with normal arch, the stenosis site had higher velocity, wall pressure and WSS, but reduced after stenosis. Patients with minor stenosis had less vortex and turbulence, usually at the site of dilation after stenosis. The impinging velocity jet could be less severe as well. For patient with angulated arch, the vortices and turbulence could be more severe and began from the arch to the distal region of the descending aorta. WSS usually got lower in the place where vortex occurred, but in the vortices with higher velocity, the pressure and WSS would turn higher.

Conclusion: With more severe deformation of the big arteries, the hemodynamic change of blood flow was more obvious.

Key Words: coarctation of aorta; computational fluid dynamics; magnetic resonance imaging; wall shear stress; cardiac surgery; artery geometry

\section{INTRODUCTION}

Coarctation of aorta $(\mathrm{CoA})$ is the most common congenital heart defect, with the incidence rate of about $9 \%$ of congenital heart defect annually. ${ }^{1}$ With surgery or intervention treatment at an early age, patients usually have good prognosis of low mortality and normal cardiac function..$^{2-4}$ Unfortunately, long term morbidity for this population remains higher than normal population, due to the following complications: restenosis, aneurysm formation and hypertension. ${ }^{5}$ Some researches have postulated that altered vascular structure would lead to hemodynamic changes and aggravate the damage of vascular wall, but the concrete mechanisms remain unclear. ${ }^{6,7}$ How to evaluate the hemodynamic abnormality quantitatively is also a challenge.

Computational fluid dynamics (CFD) technique has been conducted in the hemodynamic assessment for great arteries successfully in a series of studies. Based on the anatomic data obtained from routine CT or MR images, CFD can be used to extract 3-dimensional subject-specific anatomy of the target vessels, and simulate the vascular biomechanism with multiple parameters. ${ }^{8}$

This is an open access journal, and articles are distributed under the terms of the Creative Commons Attribution-NonCommercial-ShareAlike 4.0 License, which allows others to remix, tweak, and build upon the work non-commercially, as long as appropriate credit is given and the new creations are licensed under the identical terms.

For reprints contact: weda-h@weda-h.org

How to cite this article: Yao Q, Pan LL, Shen QL, Qiao ZW, Ridderbos FS, Hu XH. Comparison of hemodynamics in post-operation coarctation of aorta patients with different aortic arch geometry. J ADV HEALTH 2019; 1(3): 211-215. 
Although this approach has been applied to Glenn, Fontan procedure, as well as CoA surgery, the link between altered indices and different types of aortic deformation is still elusive. ${ }^{9,10}$

The objective of this work is to quantify the hemodynamic changes with different vascular structures of post-operation CoA, which contributes to further understanding of the pathologic effect for abnormal hemodynamics and identifying patients that need further treatment in the follow-up.

\section{METHODS}

\section{Demographics}

Four patients were included, all experienced end-end anastomosis surgery for $\mathrm{CoA}$ at the infantile period. The averaged surgery age was $4.66 \pm 3.17$ month-old and averaged age for MRI was 104.45 \pm 13.77 month-old. All the patients were male. The institutional review board approved the study and informed consent was obtained from all the patients' guardians.

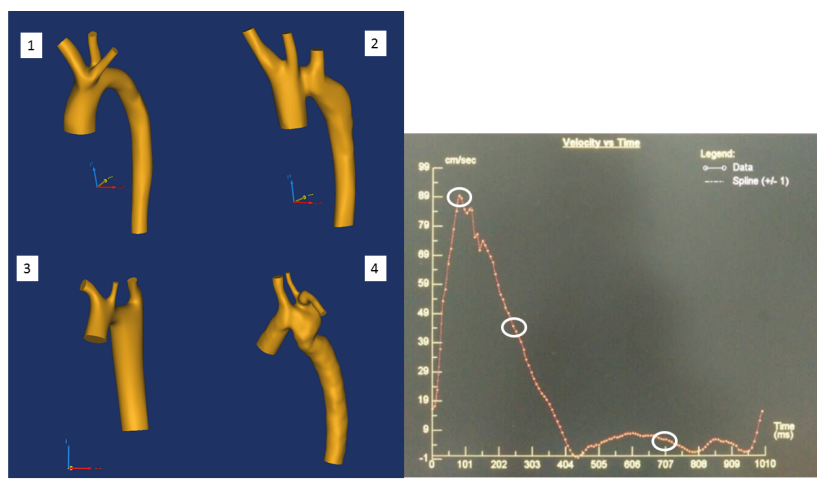

Figure 1. 3D simulated model of MRA data from four patients. Velocitytime curve was produced by MRI PC sequence for inlet flow setting, measured in end-systolic phase, middle-diastolic phase and end diastolic phase.

\section{MR and phase-contrast (PC) sequence}

Oral sedation was administered and saline-filled intra-venous access was inserted into the vein for contrast injection. A dose $(0.2$ $\mathrm{mmol} / \mathrm{kg}$ ) of gadolinium (Gd; Omniscan gadodiamide; GE Healthcare, Princeton, NJ) was injected at the rate of $2 \mathrm{ml} / \mathrm{sec}$. All the patients underwent anatomic MR and PC sequence for CFD modeling. MR was performed on a $1.5 \mathrm{~T}$ MR (Avanto, Siemens, Erlangen, Germany). Participants were instructed to hold their breath during the MR data acquisition period when age and ability permitted.

Blood flow information for CFD was acquired by using a retrospectively electrocardiogram-gated free-breathing PC sequence, with typical imaging parameters as follows: repetition time /echo time, $30 / 2 \mathrm{msec}$; flip angle, $30^{\circ}$; section thickness, $5 \mathrm{~mm}$. Planes were set $1 \mathrm{~cm}$ upper ascending aorta root and perpendicular to the flow. Velocity encoding was varied in $200-250 \mathrm{~cm} / \mathrm{s}$ according to the vessels. ${ }^{11}$

\section{CFD simulation}

Every simulation took about 6-8 hours. Rough segmentation of the aorta and 3-dimensional models for CFD simulation of the aorta and the branches were then created using Mimics 15.0 (Materialise Co., Belgium) by inputting patient-specific MR data for volume visualization. Simulations were performed using a novel stabilized finite element method to solve the conservation of mass (continuity) and balance of fluid momentum (Navier-Stokes). Vessels were assumed as rigid and blood to be a Newtonian fluid with a density of $1.06 \mathrm{~g} / \mathrm{cm}^{3}$ and a viscosity coefficient of $0.004 \mathrm{~N} / \mathrm{m}^{2} \mathrm{~s}$. Computational meshes contained more than 4 million tetrahedral elements and localized refinement was performed. After that, files were put into Fluent 15.0 (Ansys, Canonsburg) for simulations for 3-5 cardiac cycles until the flow rate yielded periodic solutions. Results for blood flow streamline, wall pressure (WP) and WSS were measured in three-phase (end-systolic phase, middle-diastolic phase and end-diastolic phase), through the ascending aorta (aAo), aortic arch (AA), as well as descending aorta (dAo) till the diaphragmatic level. The blood flow streamlines, color-coded pressure field and detailed local WSS maps were demonstrated and compared among the four patients.

\section{Inflow Boundary Conditions}

Velocity-time curve from PC sequence was used for CFD as inlet setting, using a temporally varying parabolic curve. Flow to branches was estimated from a known relationship between their diameters and aAo?

\section{RESULTS}

\section{Anatomic features}

Maximum intensity projections of the MR data were got for the four patients. No.1=no stenosis with normal "Romanesque" arch geometry, acting like normal patient; No.2=slight stenosis at the site of anastomose (stenosis/descending $=78.3 \%$ ); No.3=moderate to severe stenosis (stenosis/descending $=55.7 \%$ ) and No.4= angular (Gothic) aortic arch with slight stenosis, which meant the existence of tortuosity and augment of the AA. A region of post-stenotic dilation was consistently observed for No.2, No.3 and No.4.

CFD simulation results

Anatomic and hemodynamic assessments were captured in CFD simulations. Blood flow streamlines, WP and WSS were displayed for each patient in three phases.

Streamlines during the three phases were shown in Fig. 2. The first patient presented advection mainly and some small vortex at the sites of bifurcation. The flow pattern in No.2 and No.3 were different from the first one. We could found vortices beginning from the dilation section after the stenosis and extending along the dAo, especially in the diastolic phase. The velocity distribution was unequal. Peak systolic velocity was generally parabolic throughout the stenosis with values reaching to $2.29 \mathrm{~m} / \mathrm{s}$ (No.2) and $2.95 \mathrm{~m} / \mathrm{s}$ (No.3). The No.3 patient had more severe turbulence through the whole aorta, including big vortex and regurgitation affecting long distance of dAo. The restenosis also introduced a velocity jet that impinged on the downstream posterior wall in No2 and No.3. As for the No.4 patient with angulated AA, blood acceleration across the distorted region produced complex downstream swirling and recirculation. The vortex was even more severe through all dAo and reached higher velocity.

Simulation WP was higher in the ascending aorta and the bifurcation, for No.1 patient. Decreased WP gradients could be found through the AA and the dAo. The posterior of the vessel 


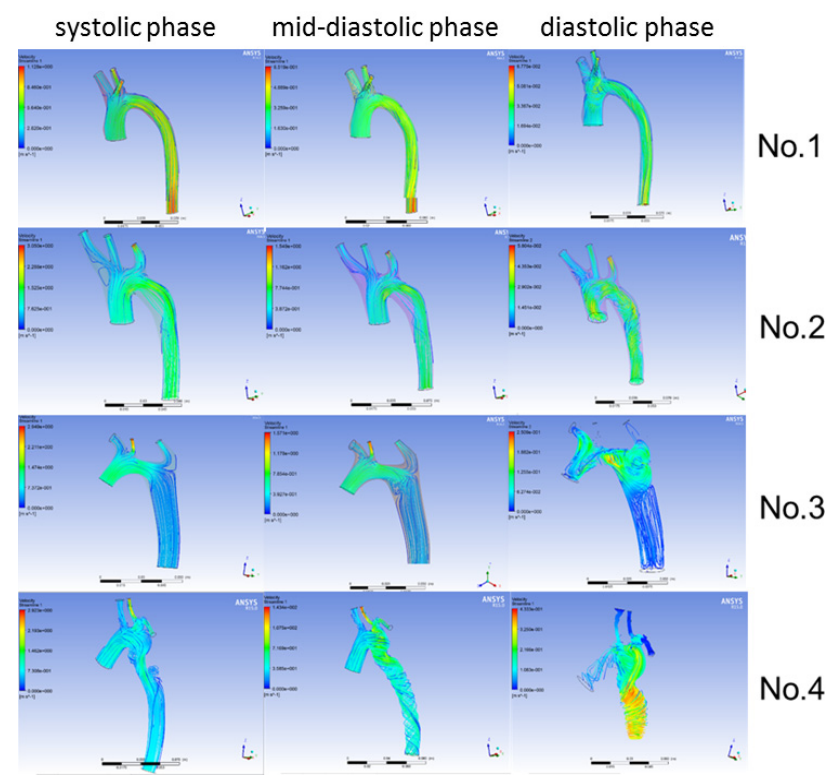

Figure 2 Blood flow streamline map from four patients in end-systolic phase, middle-diastolic phase and end diastolic phase.

usually had higher pressure than the anterior part. For No.2 patient, the similar pattern of distribution could also be found, and the WP also increased at the stenosis (1606Pa in the systolic and 55.98Pa in the diastolic). For the No.3 patient, systolic WP increased in the back part of the dilated section after the stenosis, reaching to $605 \mathrm{~Pa}$ in the systolic and decreasing most in the diastolic phase (22.58Pa). Substantial increases in systolic WP were also observed in No.4 patient, however, appearing in the aAo and AA (702.6Pa). And also, this part dropped a lot in the diastolic phase $(11 \mathrm{~Pa})$, on the contrary, pressure through the dAo got higher. The peak WP in the dilated part was located in the ventral part, according to the jet angle changed.

WSS distributions for the three patients were consistent with the flow patterns mentioned above (Fig.3). Narrowed aortic regions were generally exposed to higher WSS, which were more pronounced for the severe CoA patient (28.6 in systolic phase) than the slight patient (9.79 in systolic phase). Low WSS usually appeared in the region of bifurcation and turbulence. However, for No.3 and No.4 patients, the dilated part and the AA demonstrated higher WSS in the diastolic phase according to the higher WP and velocity inside the big vortex.

\section{DISCUSSION}

Despite CoA patients may be resolved through a number of surgical approaches, long-term morbidity still will be developed, the most notable of which are restenosis and hypertension. ${ }^{11}$ Eccentric hemodynamics reduced arterial compliance, gene expression abnormality and renin-angiotensin system activity may all contribute to this situation. ${ }^{12-14}$

Different local vascular geometry can be revealed in treated CoA patients, including stenosis, angulated arch, augmentation, shortening and high strain. ${ }^{15,16}$ It has been postulated that the aorta and its branches will adapt to maintain appropriate vascular wall stress by vascular remodeling, including thickening through the internal and medial lamellar, changing the caliber and adjusting the compliance. ${ }^{7,17}$ However, the local morphology and image-based quantification presented in this work suggested that

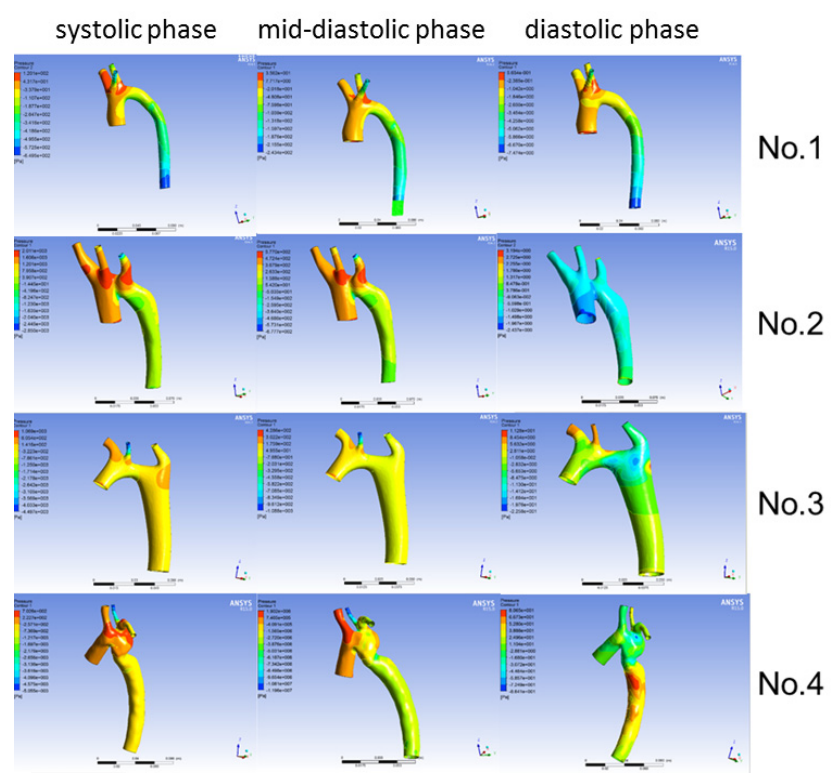

Figure 3 Wall pressure of four patients in end-systolic phase, middlediastolic phase and end diastolic phase.

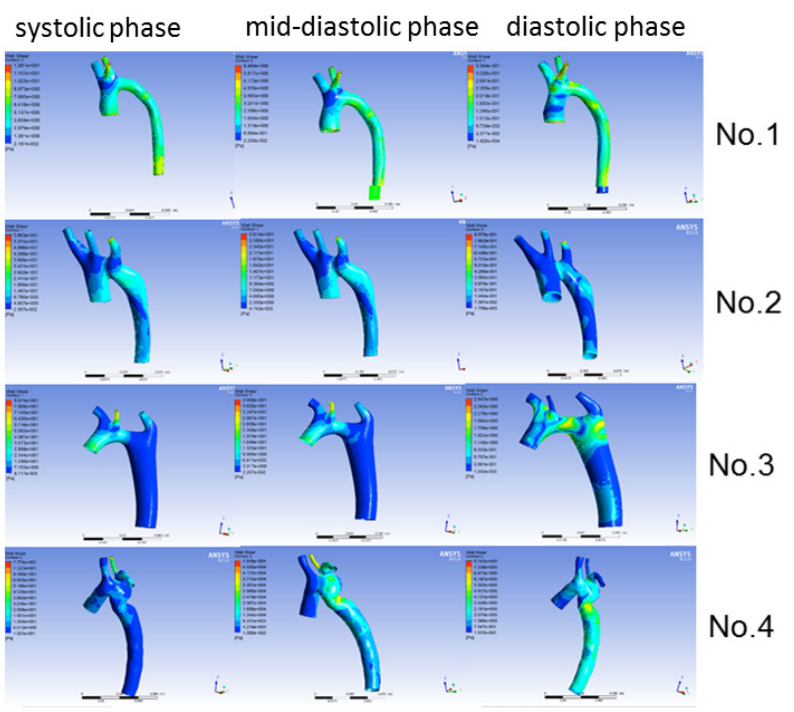

Figure 4 WSS of four patients end-systolic phase, mid-diastolic phase and end diastolic phase.

the morphology abnormality might play a key role in the hemodynamic alteration.

The current investigation introduced a coupled computational and imaging technique. CFD is a useful tool to assess detailed hemodynamic and biomechanic ramifications as well as to facilitate surgery planning through virtual surgery. ${ }^{18,19}$ The method is based on data obtained from routine clinical exams and used in combination with CFD software to generate temporally and spatially varying parameters that are hard to obtain through most of the conventional techniques available, such as digital subtraction angiography or cardiac echo.

In our research, CFD was used to obtain detailed spatiotemporal quantification of flow, the pressure to the vascular wall and WSS in the aAo, AA and dAo, offering several key findings in the following. In normal patients' flow patterns attached to the luminal surface, multiple and small helical flows would develop in the bifurcation 
area. However, these patterns altered according to the aggravated restenosis and deformation with more and bigger helical flows appearing spiraling along the descending aorta with multiple turbulence and regurgitation, usually more obvious in the diastolic phase. ${ }^{20}$ The angulated and augmented arch would present more severe turbulence with border scope. Severe stenosis would have higher peak systolic velocity values, likely due to the small dimension in this region, and produce jet flow with higher pressure to the vascular wall.

In several studies, areas of low WSS were proved to be found in areas of turbulence, and these sites are correlated with those prone to atherosclerotic plaque development. ${ }^{21,22}$ The general patterns of WSS reported in our research corresponded well with the available data. However, inside the big vortex for severely deformed vessels, higher velocity and heterogeneity usually appear, resulting in higher WSS and WP, due to the striking of the flow. The impinging jet through the severe stenosis would also lead to higher WSS and WP at the vascular wall struck. Meanwhile, at the site of stenosis with higher velocity, WSS and WP also showed higher values according to the degree of stenosis. In fact, abnormal WSS, including higher and lower values, are all correlated to vascular wall injuring and arteriosclerosis process. $^{23}$

Our research had several limitations. Only four patients were included and the relationship with clinical data was not discussed. Many important technical settings should be considered in the CFD procedure, including realistic outflow boundary conditions, fluid-structure interaction, elastic vascular wall setting, and a number of additional technical advancements in CFD. ${ }^{24,25}$ Although comparisons between patients were reported here, further studies should be conducted for the biological effect of hemodynamic force. Nonetheless, the results of our study are exciting and will likely lead to future investigations.

\section{CONCLUSION}

In summary, we reported the details characteristics of dynamic alterations with different geometry of post-operation CoA patients. The CFD is highly reproducible and combines clinical imaging data and novel computational modeling tools. Treated CoA patients are likely exposed to abnormal flow patterns, which may influence the function of the vascular wall. Thus, the current approach may be used to determine sites of atherosclerotic susceptibility before clinical apparent.

\section{Acknowledgement}

Qiong Yao and Lili Pan contributed equally to the work.

\section{Funding}

the National Key Research and Development Program of China (2016YFC1000500)

\section{Conflict of Interest}

The authors declare that they have no conflict of interest.

\section{Ethical approval}

All procedures performed in studies involving human participants were in accordance with the ethical standards of the institutional and/or national research committee and with the 1964 Helsinki declaration and its later amendments or comparable ethical standards.

\section{Informed consent}

Informed consent was obtained from all individual participants included in the study.

\section{REFERENCES}

1. Usman $\mathrm{MH}$, Rengifo-Moreno $\mathrm{P}$, Janzer SF, Inglessis-Azuaje I, Witzke-Sanz C. Coarctation of the aorta: management, indications for intervention, and advances in care. Curr Treat Options Cardiovasc Med 2014; 16(10): 341.

2. Ostovan MA, Kojuri J, Mokhtaryan M, Razazi V, Zolghadrasli A. Procedural outcome and one year follow up of patients undergoing endovascular stenting for coarctation of aorta: a single center study. $J$ Cardiovasc Thorac Res 2014; 6(2): 117-121.

3. Kim AI, Metlin SN, Ermilova NA. Outcomes after one-stage repair of coarctation of the aorta and combined intracardiac lesions in infants. Vestn Ross Akad Med Nauk 2014; (5-6): 47-51.

4. Seo DM, Park J, Goo HW, Kim YH, Ko JK, Jhang WK. Surgical modification for preventing a gothic arch after aortic arch repair without the use of foreign material. Interact Cardiovasc Thorac Surg 2015; 20(4): 504-509.

5. Lemaire A, Cuttone F, Desgué J, Ivascau C, Caprio S, Saplacan V, Belin A, Babatasi G. Late complication after repair of aortic coarctation. Asian Cardiovasc Thorac Ann 2015; 23(4): 423-429.

6. Donazzan L, Crepaz R, Stuefer J, Stellin G. Abnormalities of aortic arch shape, central aortic flow dynamics, and distensibility predispose to hypertension after successful repair of aortic coarctation. World J Pediatr Congenit Heart Surg 2014; 5(4): 546-553.

7. Menon A, Eddinger TJ, Wang H, Wendell DC, Toth JM, LaDisa JF Jr. Altered hemodynamics, endothelial function, and protein expression occur with aortic coarctation and persist after repair. Am J Physiol Heart Circ Physiol 2012; 303(11): H1304-1318.

8. LaDisa JF Jr, Dholakia RJ, Figueroa CA, Vignon-Clementel IE, Chan FP, Samyn MM, Cava JR, Taylor CA, Feinstein JA. Computational simulations demonstrate altered wall shear stress in aortic coarctation patients treated by resection with end-to-end anastomosis. Congenit Heart Dis 2011; 6(5): 432-443.

9. Restrepo M, Crouch AC, Haggerty CM, Rossignac J, Slesnick TC, Kanter KR, Yoganathan AP. Hemodynamic impact of superior vena cava placement in the Y-Graft Fontan connection. Ann Thorac Surg 2016; 101(1): 183-189.

10. Sun Q, Wan D, Liu J, Hong H, Liu Y, Zhu M. Patient-specific computational fluid dynamic simulation of a bilateral bidirectional Glenn connection. Med Biol Eng Comput 2008; 46(11): 1153-1159.

11. Chen SS, Dimopoulos K, Alonso-Gonzalez R, Liodakis E, Teijeira-Fernandez E, Alvarez-Barredo M, Kempny A, Diller G, Uebing A, Shore D, Swan L, Kilner PJ, Gatzoulis MA, Mohiaddin RH. Prevalence and prognostic implication of restenosis or dilatation at the aortic coarctation repair site assessed by cardiovascular MRI in adult patients late after coarctation repair. Int J Cardiol 2014; 173(2): 209-215.

12. Lurz P, Okon T, Riede T, Wagner R, Schuler G, Daehnert I, Desch S. Renal sympathetic denervation in uncontrolled arterial hypertension after successful repair for aortic coarctation. Int J Cardiol 2015; 202 322-327.

13. Lombardi KC, Northrup V, McNamara RL, Sugeng L, Weismann CG. 
Aortic stiffness and left ventricular diastolic function in children following early repair of aortic coarctation. Am J Cardiol 2013; 112(11): 1828-1833.

14. LaDisa JF Jr, Bozdag S, Olson J, Ramchandran R, Kersten JR, Eddinger TJ. Gene expression in experimental aortic coarctation and repair: candidate genes for therapeutic intervention? PLoS One 2015; 10(7): e0133356.

15. Mishima A, Nomura N, Ukai T, Asano M. Aortic coarctation repair in neonates with intracardiac defects: the importance of preservation of the lesser curvature of the aortic arch. J Card Surg 2014; 29(5): 692-697.

16. Liu JY, Jones B, Cheung MM, Galati JC, Koleff J, Konstantinov IE, Grigg LE, Brizard CP, d'Udekem Y. Favourable anatomy after end-to-side repair of interrupted aortic arch. Heart Lung Circ 2014; 23(3): 256-264.

17. Hayenga HN, Hu JJ, Meyer CA, Wilson E, Hein TW, Kuo L Humphrey JD. Differential progressive remodeling of coronary and cerebral arteries and arterioles in an aortic coarctation model of hypertension. Front Physiol 2012; 3: 420.

18. Sun Q, Liu J, Qian Y, Zhang H, Wang Q, Sun Y, Hong H, Liu J. Computational haemodynamic analysis of patient-specific virtual operations for total cavopulmonary connection with dual superior venae cavae. Eur J Cardiothorac Surg 2014; 45(3): 564-569.

19. Haggerty CM, de Zélicourt DA, Restrepo M, Rossignac J, Spray TL, Kanter KR, Fogel MA, Yoganathan AP. Comparing pre- and postoperative Fontan hemodynamic simulations: implications for the reliability of surgical planning. Ann Biomed Eng 2012; 40(12): 2639-2651.

20. Goubergrits L, Mevert R, Yevtushenko P, Schaller J, Kertzscher U, Meier S Schubert S, Riesenkampff E, Kuehne T. The impact of MRI-based inflow for the hemodynamic evaluation of aortic coarctation. Ann Biomed Eng 2013; 41(12): 2575-2587.

21. Choi G, Lee JM, Kim HJ, Park JB, Sankaran S, Otake H, Doh JH, Nam CW, Shin ES, Taylor CA, Koo BK. Coronary artery axial plaque stress and its relationship with lesion geometry: application of computational fluid dynamics to coronary CT angiography. JACC Cardiovasc Imaging 2015; 8(10): 1156-1166.

22. Rashdan NA, Lloyd PG. Fluid shear stress upregulates placental growth factor in the vessel wall via NADPH oxidase 4. Am J Physiol Heart Circ Physiol 2015; 309(10): H1655-1666.

23. Samady H, Eshtehardi P, McDaniel MC, Suo J, Dhawan SS, Maynard C, Timmins LH, Quyyumi AA, Giddens DP. Coronary artery wall shear stress is associated with progression and transformation of atherosclerotic plaque and arterial remodeling in patients with coronary artery disease. Circulation 2011; 124(7): 779-788.

24. Selvarasu NK, AAfti DK. Effects of elastic modulus change in helical tubes under the influence of dynamic changes in curvature and torsion. J Biomech Eng 2014; 136(8).

25. Morbiducci U, Gallo D, Massai D, Consolo F, Ponzini R, Antiga L, Bignardi C, Deriu MA, Redaelli A. Outflow conditions for image-based hemodynamic models of the carotid bifurcation: implications for indicators of abnormal flow. J Biomech Eng 2010; 132(9): 091005. 